\title{
Distribution of crossings, nestings and alignments of two edges in matchings and partitions
}

\author{
Anisse Kasraoui and Jiang Zeng \\ Institut Camille Jordan \\ Université Claude Bernard (Lyon I) \\ F-69622, Villeurbanne Cedex, France \\ anisse@math.univ-lyon1.fr, zeng@math.univ-lyon1.fr \\ Submitted: Nov 11, 2005; Accepted: Mar 30, 2006; Published: Apr 4, 2006 \\ Mathematics Subject Classifications: 05A18; 05A15, 05A30
}

\begin{abstract}
We construct an involution on set partitions which keeps track of the numbers of crossings, nestings and alignments of two edges. We derive then the symmetric distribution of the numbers of crossings and nestings in partitions, which generalizes a recent result of Klazar and Noy in perfect matchings. By factorizing our involution through bijections between set partitions and some path diagrams we obtain the continued fraction expansions of the corresponding ordinary generating functions.
\end{abstract}

\section{Introduction}

A partition of $[n]:=\{1,2, \cdots, n\}$ is a collection of disjoint nonempty subsets of $[n]$, called blocks, whose union is $[n]$. A (perfect) matching of $[2 n]$ is a partition of $[2 n]$ in $n$ twoelement blocks. The set of partitions (resp. matchings) of $[n]$ will be denoted by $\Pi_{n}$ (resp. $\mathcal{M}_{n}$ ). A standard way of writing a partition $\pi$ with $k$ blocks is $\pi=B_{1}-B_{2}-\cdots-B_{k}$, where the blocks are ordered in the increasing order of their minimum elements and, within each block, the elements are written in the numerical order.

It is convenient to identify a partition of $[n]$ with a partition graph on the vertex set $[n]$ such that there is an edge joining $i$ and $j$ if and only if $i$ and $j$ are consecutive elements in a same block. We note such an edge $e$ as a pair $(i, j)$ with $i<j$, and say that $i$ is the left-hand endpoint of $e$ and $j$ is the right-hand endpoint of $e$. A singleton is the element of a block which has only one element, so a singleton corresponds to an isolated vertex in the graph. Conversely, a graph on the vertex set $[n]$ is a partition graph if and only if each vertex is the left-hand (resp. right-hand) endpoint of at most one edge. By convention, the vertices $1,2, \cdots, n$ are arranged on a line in the increasing order from left to right and an edge $(i, j)$ is drawn as an arc above the line. An illustration is given in Figure 1. 


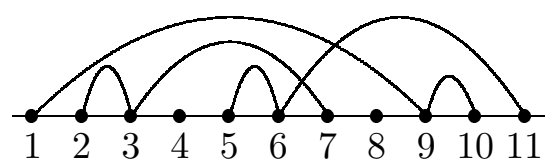

Figure 1: Graph of the partition $\pi=\{1,9,10\}-\{2,3,7\}-\{4\}-\{5,6,11\}-\{8\}$

Given a partition $\pi$ of $[n]$, two edges $e_{1}=\left(i_{1}, j_{1}\right)$ and $e_{2}=\left(i_{2}, j_{2}\right)$ of $\pi$ are said to form:

(i) a crossing with $e_{1}$ as the initial edge if $i_{1}<i_{2}<j_{1}<j_{2}$;

(ii) a nesting with $e_{2}$ as interior edge if $i_{1}<i_{2}<j_{2}<j_{1}$;

(iii) an alignment with $e_{1}$ as initial edge if $i_{1}<j_{1} \leq i_{2}<j_{2}$.

An illustration of these notions is given in Figure 2.

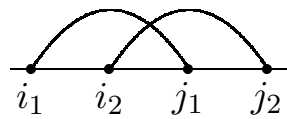

$(i)$

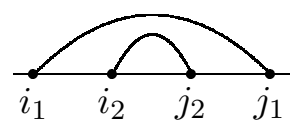

(ii)

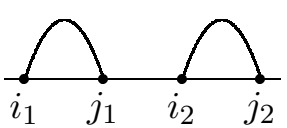

(iii)

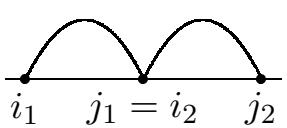

$\left(i i i^{\prime}\right)$

Figure 2: Crossing, nesting and alignments of two edges

The numbers of crossings, nestings and alignments of two edges in $\pi$ will be denoted by $\operatorname{cr}(\pi)$, ne $(\pi)$ and $\operatorname{al}(\pi)$, respectively. For the partition $\pi$ in Figure 1, we have $\operatorname{cr}(\pi)=2$, $\operatorname{ne}(\pi)=5$ and $\operatorname{al}(\pi)=8$.

Now, assume that $B$ is a partition block with at least two elements. Then an element of $B$ is said to be:

(i) an opener if it is the least element of $B$,

(ii) a closer if it is the greatest element of $B$,

(iii) a transient if it is neither the least nor greatest elements of $B$.

In a partition graph, the edges around an opener, closer, singleton or transient look as follows:
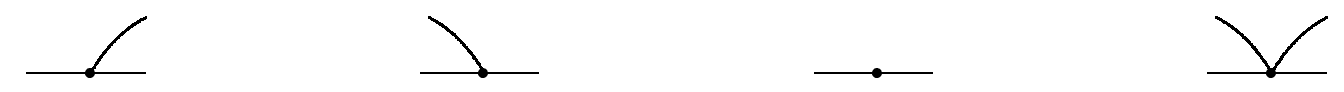

Figure 3: Opener, closer, singleton and transient in a partition graph

The sets of openers, closers, singletons and transients of $\pi$ will be denoted by $\mathcal{O}(\pi)$, $\mathcal{C}(\pi), \mathcal{S}(\pi)$ and $\mathcal{T}(\pi)$, respectively. The 4-tuple $\lambda(\pi)=(\mathcal{O}(\pi), \mathcal{C}(\pi), \mathcal{S}(\pi), \mathcal{T}(\pi))$ is called the type of $\pi$. For example, for the partition $\pi$ in Figure 1, we have $\mathcal{O}(\pi)=\{1,2,5\}$, $\mathcal{C}(\pi)=\{7,10,11\}, \mathcal{S}(\pi)=\{4,8\}$ and $\mathcal{T}(\pi)=\{3,6,9\}$. 
Definition 1.1 A 4-tuple $\lambda=(\mathcal{O}, \mathcal{C}, \mathcal{S}, \mathcal{T})$ of subsets of $[n]$ is a partition type of $[n]$ if there exists a partition of $[n]$ whose type is $\lambda$. Denote by $\Pi_{n}(\lambda)$ the set of partitions of type $\lambda$, i.e.,

$$
\Pi_{n}(\lambda)=\left\{\pi \in \Pi_{n}: \lambda(\pi)=\lambda\right\} .
$$

In particular, a partition type $\lambda$ is a matching type if $\lambda=(\mathcal{O}, \mathcal{C}):=(\mathcal{O}, \mathcal{C}, \emptyset, \emptyset)$. Denote by $\mathcal{M}_{2 n}(\gamma)$ the set of matchings of type $\gamma$, i.e.,

$$
\mathcal{M}_{2 n}(\gamma)=\left\{\alpha \in \mathcal{M}_{2 n}: \mathcal{O}(\alpha)=\mathcal{O} \text { and } \mathcal{C}(\alpha)=\mathcal{C}\right\} .
$$

Our main result is the construction of an explicit involution on the set of partitions and is stated in the following

Theorem 1.2 There is an involution $\varphi: \Pi_{n} \rightarrow \Pi_{n}$ preserving the type of partitions and number of alignments, and exchanging the numbers of crossings and nestings. In other words, for each $\pi \in \Pi_{n}$, we have $\lambda(\pi)=\lambda(\varphi(\pi))$ and

$$
\operatorname{al}(\varphi(\pi))=\operatorname{al}(\pi), \operatorname{cr}(\varphi(\pi))=\operatorname{ne}(\pi), \operatorname{ne}(\varphi(\pi))=\operatorname{cr}(\pi) .
$$

We derive immediately the following equality of the corresponding generating functions.

Corollary 1.3 For each partition type $\lambda$ of $[n]$, we have

$$
\sum_{\pi \in \Pi_{n}(\lambda)} p^{\operatorname{cr}(\pi)} q^{\mathrm{ne}(\pi)} t^{\mathrm{al}(\pi)}=\sum_{\pi \in \Pi_{n}(\lambda)} p^{\mathrm{ne}(\pi)} q^{\operatorname{cr}(\pi)} t^{\mathrm{al}(\pi)},
$$

and for each matching type $\gamma$ of $[2 n]$,

$$
\sum_{\alpha \in \mathcal{M}_{2 n}(\gamma)} p^{\operatorname{cr}(\alpha)} q^{\operatorname{ne}(\alpha)} t^{\operatorname{al}(\alpha)}=\sum_{\alpha \in \mathcal{M}_{2 n}(\gamma)} p^{\operatorname{ne}(\alpha)} q^{\operatorname{cr}(\alpha)} t^{\operatorname{al}(\alpha)} .
$$

Summing over all partition types $\lambda$ or matching types $\gamma$ we get

\section{Corollary 1.4}

$$
\sum_{\pi \in \Pi_{n}} p^{c r(\pi)} q^{n e(\pi)} t^{a l(\pi)}=\sum_{\pi \in \Pi_{n}} p^{n e(\pi)} q^{c r(\pi)} t^{a l(\pi)},
$$

and

$$
\sum_{\alpha \in \mathcal{M}_{2 n}} p^{\operatorname{cr}(\alpha)} q^{\mathrm{ne}(\alpha)} t^{\mathrm{al}(\alpha)}=\sum_{\alpha \in \mathcal{M}_{2 n}} p^{\mathrm{ne}(\alpha)} q^{\operatorname{cr}(\alpha)} t^{\mathrm{al}(\alpha)} .
$$

By specializing $t=1$ in the above corollary, we obtain

\section{Corollary 1.5}

$$
\sum_{\pi \in \Pi_{n}} p^{c r(\pi)} q^{n e(\pi)}=\sum_{P \in \Pi_{n}} p^{n e(\pi)} q^{c r(\pi)},
$$

and

$$
\sum_{\alpha \in \mathcal{M}_{2 n}} p^{\operatorname{cr}(\alpha)} q^{\operatorname{ne}(\alpha)}=\sum_{\alpha \in \mathcal{M}_{2 n}} p^{\operatorname{ne}(\alpha)} q^{\operatorname{cr}(\alpha)} .
$$


Klazar [8] has given a different proof of (1.7) and pointed out that equation (1.7) was first obtained by Klazar and Noy. Previously the special $p=1$ case of (1.7) had been proved by De Sainte-Catherine [5] and also by De Médicis and Viennot [4].

We were inspired by the general combinatorial theory of orthogonal polynomials and continued fractions developed by Viennot [14] and Flajolet [6]. In fact, our involution $\varphi$ is a direct generalization of that used by De Médicis and Viennot [4] for matchings. A variant of this bijection on partitions has been used by Ksavrelof and Zeng [10] to prove other equinumerous results on partitions.

The rest of this paper is organized as follows: we shall present the involution $\varphi$ and the proof of theorem 1.1 in section 2 ; in section 3 we factorize our involution through two bijections $\varphi_{l}$ and $\varphi_{r}$ between partitions and Charlier diagrams; in section 4, we apply $\varphi_{l}$ or $\varphi_{r}$ to derive continued fraction expansions of the ordinary generating functions with respect to the numbers of crossings and nestings of two edges in matchings and partitions.

\section{Proof of Theorem 1.2}

Let $\pi=B_{1}-B_{2}-\cdots-B_{k}$ be a partition of $[n]$ and $i$ an integer in $[n]$. The $i$-th trace of $\pi$ is defined by

$$
T_{i}(\pi)=B_{1}(\leq i)-B_{2}(\leq i)-\cdots-B_{k}(\leq i),
$$

where $B_{j}(\leq i):=B_{j} \cap[i]$ is the restriction of the block $B_{j}$ on $[i]$. One says that $B_{j}(\leq i)$ is active (resp. closed and empty) if $B_{j} \nsubseteq[i]$ (resp. $B_{j} \subseteq[i]$ and $B_{j} \cap[i]=\emptyset$ ).

We shall identify $T_{i}(\pi)$ with the subgraph $D_{i}(\pi)$ of the graph $D(\pi)$ induced by the vertex set $[i]$, with the additional condition that for any edge $(x, y)$ of $\pi$ such that $x \leq$ $i<y$, we attach a "half-edge" to the vertex $x$, called vacant vertex. Denote by $l_{i}(\pi)$ the number of vacant vertices in $D_{i-1}(\pi)$, with $D_{0}=\emptyset$. Moreover, if $i$ is a closer or a transient, there is an edge $(j, i)$ with $j<i$, we denote by $\gamma_{i}(\pi)$ the rank of the vertex $j$ among the vacant vertices of $D_{i-1}(\pi)$, the vacant vertices being arranged from left to right in the order of their creation, namely, in increasing order.

For instance, if $\pi$ is the partition given in Figure 1, then

$$
\begin{aligned}
& T_{5}(\pi)=\{1 .\}-\{2,3 .\}-\{4\}-\{5 .\}, \\
& T_{6}(\pi)=\{1 .\}-\{2,3 .\}-\{4\}-\{5,6 .\},
\end{aligned}
$$

where each active block is ended with a dot. Hence $l_{6}(\pi)=3$ and $\gamma_{6}(\pi)=3$. The corresponding graphs $D_{5}(\pi)$ and $D_{6}(\pi)$ are given as follows:

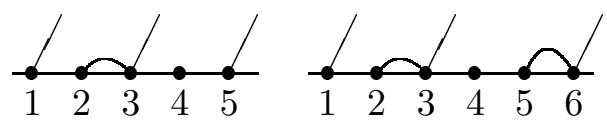


Now, we can describe our fundamental bijection $\varphi$ on $\Pi_{n}$ using the graphs of traces. In the following, by "declare the vertex $i$ vacant" we mean "attach a half-edge to the vertex $i$ ". Let $\pi \in \Pi_{n}$ then $\varphi(\pi) \in \Pi_{n}$ is defined by the following algorithm:

1. Set $D_{0}^{\prime}=\emptyset$.

2. For $1 \leq i \leq n$, the graph $D_{i}^{\prime}$ is obtained from $D_{i-1}^{\prime}$ by adding $i$ as follows:

(i) if $i \in \mathcal{O}(\pi)$, declare the vertex $i$ vacant.

(ii) if $i \in \mathcal{S}(\pi)$, add $i$ as an isolated vertex.

(iii) if $i \in \mathcal{C}(\pi) \cup \mathcal{T}(\pi)$, join $i$ to the $\gamma_{i}(\pi)$-th (from right to left) vacant vertex of $D_{i-1}^{\prime}$. Moreover, if $i \in T$, declare the vertex $i$ vacant.

3. Set $\varphi(\pi):=D_{n}^{\prime}$.

For instance, if $\pi$ is as before, then the step-by-step construction of $\varphi(\pi)$ is given in Figure 4 and $\varphi(\pi)=\{1,3,10\}-\{2,6,9,11\}-\{4\}-\{5,7\}-\{8\}$. As a check, we note that $\operatorname{cr}(\varphi(\pi))=\operatorname{ne}(\pi)=5, \operatorname{ne}(\varphi(\pi))=\operatorname{cr}(\pi)=2$ and $\operatorname{al}(\varphi(\pi))=\operatorname{al}(\pi)=8$.

We shall decompose the proof of Theorem 1.1 in two lemmas.

Lemma 2.1 The mapping $\varphi: \Pi_{n} \rightarrow \Pi_{n}$ is an involution which preserves the type.

Proof. By induction on $i(0 \leq i \leq n)$, it is easy to see that $D_{i}^{\prime}$ has the same vacant vertices as $D_{i}(\pi)$. So $(i i i)$ is valid and the algorithm is well defined. By inspecting the algorithm, we see that $\varphi(\pi)$ has the same type as $\pi$. To see $\varphi$ is an involution, it suffices to notice that applying the operation "reverse the order of the vacant vertices in (iii)" twice is equal to keep the original order.

Remark 2.1 The graph $D_{i}^{\prime}$ corresponds with the graph of $i$-th trace of $\varphi(\pi)$.

To complete the proof of Theorem 1.1 it remains to verify (1.1). In fact we shall prove a stronger result. For any closer or transient $j$ of a partition $\pi$, let $\operatorname{cr}(\pi ; \mathrm{j})(\operatorname{resp} . \operatorname{ne}(\pi ; \mathrm{j})$ and $\mathrm{al}(\pi ; \mathrm{j}))$ be the number of crossings (resp. nestings and alignments) whose initial (resp. interior and initial) edge has $j$ as the right-hand endpoint. Clearly

$$
\operatorname{cr}(\pi)=\sum \operatorname{cr}(\pi ; \mathrm{j}), \quad \operatorname{ne}(\pi)=\sum \operatorname{ne}(\pi ; \mathrm{j}), \quad \operatorname{al}(\pi)=\sum \operatorname{al}(\pi ; \mathrm{j}),
$$

where the summations are over $j \in C(\pi) \cup T(\pi)$.

Lemma 2.2 Let $\pi$ be a partition of $[n]$ and $j$ a closer or transient of $\pi$. Then

$$
\operatorname{al}(\varphi(\pi) ; \mathrm{j})=\operatorname{al}(\pi ; \mathrm{j}), \quad \operatorname{cr}(\varphi(\pi) ; \mathrm{j})=\operatorname{ne}(\pi ; \mathrm{j}), \quad \operatorname{ne}(\varphi(\pi) ; \mathrm{j})=\operatorname{cr}(\pi ; \mathrm{j})
$$




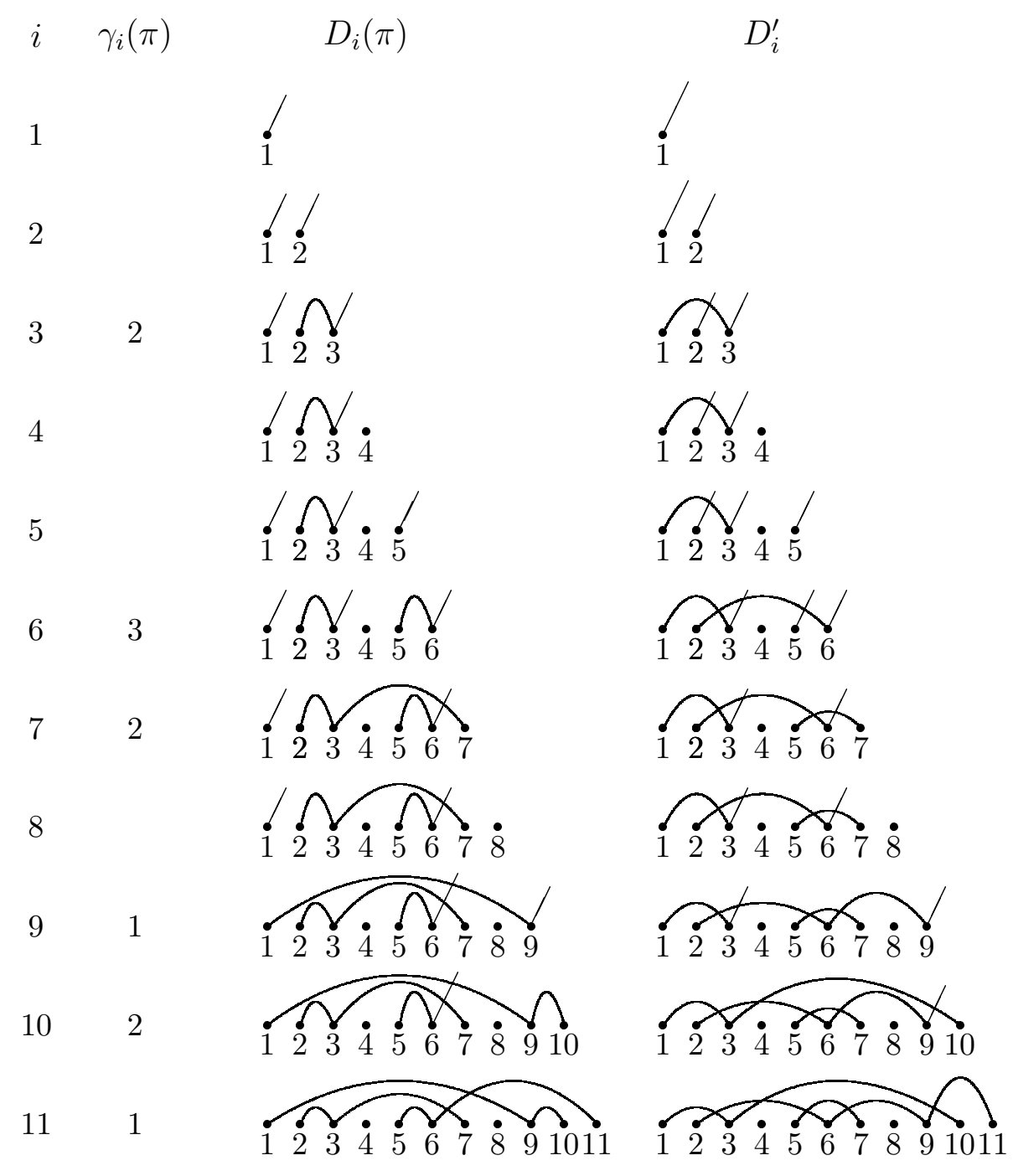

Figure 4: Construction of $\varphi(\pi)=\{1,3,10\}-\{2,6,9,11\}-\{4\}-\{5,7\}-\{8\}$

Proof. For any partition $\pi$, the number of alignments with $j$ as the right-hand endpoint, i.e. $\operatorname{al}(\pi ; \mathrm{j})$, is equal to the number of openers and transients which are $\geq j$. Now, as $\varphi(\pi)$ has the same openers and transients as $\pi$, we get immediately $\operatorname{al}(\varphi(\pi) ; \mathrm{j})=\operatorname{al}(\pi ; \mathrm{j})$.

Next, in the $j$-th $(1 \leq j \leq n-1)$ step of the construction of $\varphi(\pi)$, we add the vertex $j$ to $D_{j-1}^{\prime}$ for obtaining $D_{j}^{\prime}$. There are exactly $l_{j}:=l_{j}(\pi)$ vacant vertices in $D_{j-1}^{\prime}$ (resp. $\left.D_{j-1}\right)$. These vertices are smaller than $j$ and arranged from left to right in increasing order. Suppose that $j$ is linked with the $\gamma_{j}$-th vacant vertex $\bar{j}$ of $D_{j-1}$ in $D_{j}$ (resp. $D_{j-1}^{\prime}(\pi)$ in $\left.D_{j}^{\prime}\right)$. Recall that the rank of vacant vertices is counted from left to right in $D_{j-1}$ and from right to left in $D_{j-1}^{\prime}$.

- Any vacant vertex $\alpha$ on the left of the vertex $\bar{j}$ in $D_{j}$ (resp. $D_{j}^{\prime}$ ) will be linked to a vertex $\beta$ on the right of the vertex $j$; thus $(\alpha, \beta)$ will form a nesting with $(\bar{j}, j)$ as 

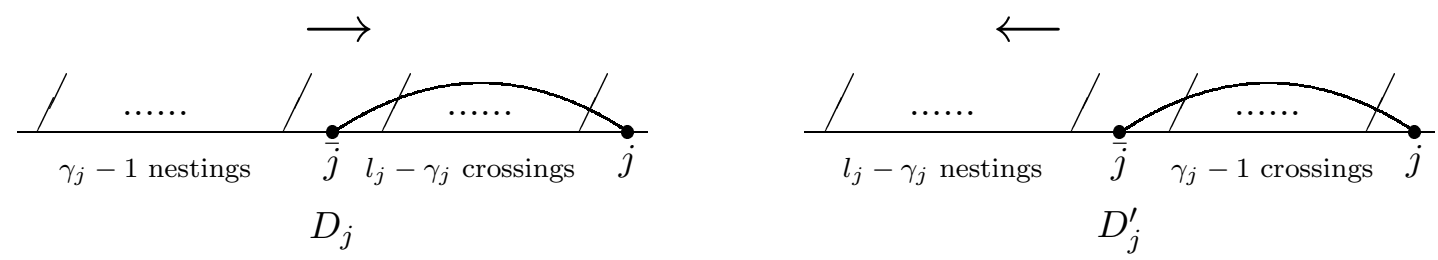

Figure 5: Counting of $\operatorname{cr}(\pi ; \mathrm{j})$ and $\operatorname{cr}(\varphi(\pi) ; \mathrm{j})$

an interior edge in $D_{n}$ (resp. $\left.D_{n}^{\prime}\right)$. Conversely, if $(a, b)$ forms a nesting with interior edge $(\bar{j}, j)$, then $a$ must be a vacant vertex on the left of the vertex $\bar{j}$ in $D_{j}$ (resp. $\left.D_{j}^{\prime}\right)$. We deduce that ne $(\pi ; \mathrm{j})=\gamma_{\mathrm{j}}-1$ and $\operatorname{ne}(\varphi(\pi) ; \mathrm{j})=\mathrm{l}_{\mathrm{j}}-\gamma_{\mathrm{j}}$.

- Any vacant vertex $\alpha$ between $\bar{j}$ and $j$ in $D_{j}$ (resp. $D_{j}^{\prime}$ ) will be linked to a vertex $\beta$ on the right of the vertex $j$; thus $(\alpha, \beta)$ will form a crossing with initial edge $(\bar{j}, j)$. Conversely, if $(a, b)$ forms a crossing with initial edge $(\bar{j}, j)$, then the vertex $a$ must be a vacant vertex on the right of the vertex $\bar{j}$ in $D_{j}$ (resp. $\left.D_{j}^{\prime}\right)$. We deduce that $\operatorname{cr}(\pi ; \mathrm{j})=\mathrm{l}_{\mathrm{j}}-\gamma_{\mathrm{j}}$ and $\operatorname{cr}(\varphi(\pi) ; \mathrm{j})=\gamma_{\mathrm{j}}-1$.

The proof is completed by comparing the above counting results.

\section{Factorization of $\varphi$ via Charlier diagrams}

\subsection{Charlier diagrams}

A path of length $n$ is a finite sequence $w=\left(s_{0}, s_{1}, \cdots, s_{n}\right)$ of points $s_{i}=\left(x_{i}, y_{i}\right)$ in the plan $\mathbb{Z} \times \mathbb{Z}$. A step $\left(s_{i}, s_{i+1}\right)$ of $w$ is East (resp. North-East and South-East) if $s_{i+1}=\left(x_{i}+1, y_{i}\right)$ (resp. $s_{i+1}=\left(x_{i}+1, y_{i}+1\right)$ and $\left.s_{i+1}=\left(x_{i}+1, y_{i}-1\right)\right)$. The number $y_{i}$ is the height of the step $\left(s_{i}, s_{i+1}\right)$. The integer $i+1$ is the index of the step $\left(s_{i}, s_{i+1}\right)$.

A Motzkin path is a path $w=\left(s_{0}, s_{1}, \cdots, s_{n}\right)$ such that: $s_{0}=(0,0), s_{n}=(n, 0)$, each step is East or North-East or South-East and the height of each step is nonnegative. A bicolored Motzkin (BM) path is a Motzkin path whose East steps are colored with red or blue. A restricted bicolored Motzkin (RBM) path is a BM path whose blue East steps are of positive height.

In the following, we shall write $B E, R E, N E$ and $S E$ as abbreviations of Blue East, Red East, North-East and South-East.

Definition 3.1 A Charlier diagram of length $n$ is a pair $h=(w, \xi)$ where $w=\left(s_{0}, \ldots, s_{n}\right)$ is a $R B M$ path and $\xi=\left(\xi_{1}, \ldots, \xi_{n}\right)$ is a sequence of integers such that $\xi_{i}=1$ if the $i$-th step is $N E$ or $R E$, and $1 \leq \xi_{i} \leq h_{i}$ if the $i$-th step is $S E$ or BE of height $h_{i}$. The set of Charlier diagrams of length $n$ is denoted by $\Gamma_{n}$.

A Charlier diagram is given in Figure 6. The type of a BM path $w$ is the 4-tuple $\lambda(w)=(\mathcal{O}(w), \mathcal{C}(w), \mathcal{S}(w), \mathcal{T}(w))$, where $\mathcal{O}(w)$ (resp. $\mathcal{C}(w), \mathcal{S}(w), \mathcal{T}(w))$ is the set of 
indices of NE (resp. SE, RE, BE) steps of $w$. For instance, if $w$ is the path in Figure 6, then

$$
\lambda(w)=(\{1,2,5\},\{7,10,11\},\{4,8\},\{3,6,9\}) .
$$

Denote by $M_{b}(n)$ (resp. $\left.M_{r b}(n)\right)$ the set of BM (resp. RBM) paths of length $n$.

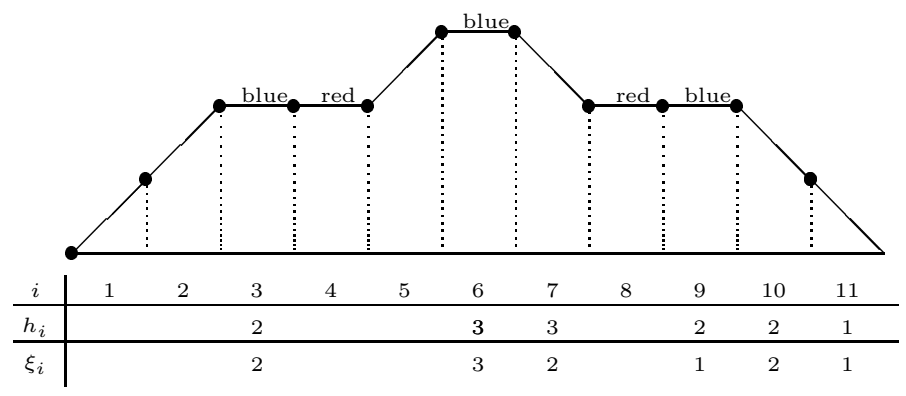

Figure 6: A Charlier diagram of length 11

There is a well-known bijection (see $[6,14]$ ) from $\Gamma_{n}$ to $\Pi_{n}$. For our purpose, we present two variants $\varphi_{l}$ and $\varphi_{r}$ of this bijection, which keep the track of crossings and nestings. The description of the bijection $\varphi_{l}\left(\operatorname{resp} . \varphi_{r}\right)$ is based on the fact that a partition $\pi$ of $[n]$ is completely determined by its type $\lambda=(\mathcal{O}, \mathcal{C}, \mathcal{S}, \mathcal{T})$ and the integers $\gamma_{i}(\pi), i \in \mathcal{C} \cup \mathcal{T}$.

Given a Charlier diagram $h=(w, \xi)$ of length $n$, we define the partition $\pi=\varphi_{l}(h)$ as follows: the type of $\pi$ is that of $w$ and $\gamma_{j}(\pi):=\xi_{j}$, for $j \in \mathcal{C} \cup \mathcal{T}$.

To define $\varphi_{l}$, we take $\xi_{j}$ as the rank from left to right of the vacant vertex linked to $j$ in the $j$-th step of the construction of $\pi$. If we take $\xi_{j}$ as the rank from right to left of the vacant vertex linked to $j$ in the $j$-th step of the construction of $\pi$, then we get the bijection $\varphi_{r}$. Hence we have the identity: $\varphi_{r}=\varphi \circ \varphi_{l}$. In other words, the following diagram is commutative:

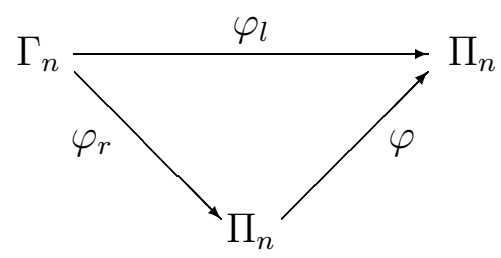

Figure 7: Factorization of $\varphi$

Denote, respectively, by $\operatorname{sg}(\pi), \operatorname{bl}(\pi)$ and $\operatorname{tr}(\pi)$ the numbers of singletons, blocks whose cardinal number is $\geq 2$ and transients of a partition $\pi$. The following result is clear (cf. $[6,14])$.

Proposition 3.2 The mappings $\varphi_{l}$ and $\varphi_{r}: \Gamma_{n} \rightarrow \Pi_{n}$ are bijections. Moreover, if $h=$ $(w, \xi) \in \Gamma_{n}$ and $\pi=\varphi_{l}(h)$ or $\varphi_{r}(h)$, then $\operatorname{sg}(\pi)$ (resp. $\operatorname{bl}(\pi)$ and $\operatorname{tr}(\pi)$ ) is equal to the number of red East (resp. North-East and blue East) steps of $w$. 
For instance, if $h=(w, \xi)$ is the Charlier diagram of Figure 6, the construction of $\varphi_{l}(h)$ (resp. $\left.\varphi_{r}(h)\right)$ corresponds with the traces sequence $D_{i}(\pi)$ (resp. $D_{i}^{\prime}$ ) in Figure 4. In other words, we have

$$
\begin{aligned}
& \varphi_{l}(h)=\{1,9,10\}-\{2,3,7\}-\{4\}-\{5,6,11\}-\{8\}, \\
& \varphi_{r}(h)=\{1,3,10\}-\{2,6,9,11\}-\{4\}-\{5,7\}-\{8\} .
\end{aligned}
$$

Proposition 3.3 Let $h=(w, \xi)$ be a Charlier diagram such that the $j$-th step of $w$ is blue East or South-East of height $k$, then

$$
\begin{aligned}
\operatorname{cr}\left(\varphi_{\mathrm{r}}(\mathrm{h}) ; \mathrm{j}\right) & =\operatorname{ne}\left(\varphi_{\mathrm{l}}(\mathrm{h}) ; \mathrm{j}\right)=\xi_{\mathrm{j}}-1 \\
\operatorname{ne}\left(\varphi_{\mathrm{r}}(\mathrm{h}) ; \mathrm{j}\right) & =\operatorname{cr}\left(\varphi_{\mathrm{l}}(\mathrm{h}) ; \mathrm{j}\right)=\mathrm{k}-\xi_{\mathrm{j}}
\end{aligned}
$$

Proof. This follows from the proof of Lemma 2.2 by replacing $\varphi_{l}(h)$ by $\pi, \varphi_{r}(h)$ by $\varphi(\pi)$, $l_{j}$ by $k$ and $\gamma_{j}$ by $\xi_{j}$.

A partition $\pi$ is noncrossing (resp. nonnesting) if $\operatorname{cr}(\pi)=0$ (resp. ne $(\pi)=0)$. Let $N C_{n}$ (resp. $N N_{n}$ ) be the set of noncrossing (resp. nonnesting) partitions of $[n]$.

Corollary 3.4 Let 1 denote the $n$-tuple $(1,1, \ldots, 1)$. Then

(i) The mapping $w \mapsto \varphi_{r}((w, \mathbf{1}))$ is a bijection from $M_{r b}(n)$ to $N C_{n}$.

(ii) The mapping $w \mapsto \varphi_{l}((w, \mathbf{1}))$ is a bijection from $M_{r b}(n)$ to $N N_{n}$.

Proof. Let $h=(w, \xi)$ a restricted diagram and suppose that the $j$-th step of $w$ is blue East or South-East. Then, Proposition 3.3 implies that $\operatorname{cr}\left(\varphi_{\mathrm{r}}(\mathrm{h}) ; \mathrm{j}\right)=\operatorname{ne}\left(\varphi_{\mathrm{l}}(\mathrm{h}) ; \mathrm{j}\right)=\xi_{\mathrm{j}}-1$. Thus the partition $\varphi_{r}(h)$ (resp. $\left.\varphi_{l}(h)\right)$ is noncrossing (resp. nonnesting) if and only if $\xi_{i}=1$ for each $i$.

Note that Corollary 3.4 gives another proof of the well-known fact (see [11] and [12, p.226]) that the numbers of elements in both $N C_{n}$ and $N N_{n}$ are equal to the $n$-th Catalan number $C_{n}=\frac{1}{n+1}\left(\begin{array}{c}2 n \\ n\end{array}\right)$. Moreover, the mapping $\varphi=\varphi_{l} \circ \varphi_{r}^{-1}: N C_{n} \rightarrow N N_{n}$ is a bijection.

\section{Continued fraction expansions}

Consider the enumerating polynomial of $\Pi_{n}$ :

$$
B_{n}\left(p, q, u_{1}, u_{2}, v\right)=\sum_{\pi \in \Pi_{n}} p^{\operatorname{cr}(\pi)} q^{\mathrm{ne}(\pi)} u_{1}^{s g(\pi)} u_{2}^{\mathrm{bl}(\pi)} v^{\operatorname{tr}(\pi)},
$$

which is a generalization of $n$-th Bell numbers. Let

$$
[n]_{p, q}=\frac{p^{n}-q^{n}}{p-q}, \quad[n]_{q}=\frac{1-q^{n}}{1-q} .
$$


It follows from Proposition 3.3 that

$$
\begin{aligned}
& B_{n}\left(p, q, u_{1}, u_{2}, v\right) \\
& =\sum_{(w, \xi) \in \Gamma_{n}}\left(\prod_{j \in \mathcal{O}(w)} u_{2}\right)\left(\prod_{j \in \mathcal{S}(w)} u_{1}\right)\left(\prod_{j \in \mathcal{C}(w)} p^{\xi_{j}-1} q^{h_{j}-\xi_{j}}\right)\left(\prod_{j \in \mathcal{T}(w)} p^{\xi_{j}-1} q^{h_{j}-\xi_{j}} v\right),
\end{aligned}
$$

where $h_{j}$ is the height of the $j$-th step of $w$.

We can rewrite the double sums in (4.1) as a single sum on bicolored Motzkin paths. For any BM path $w$, define the weight of a step of $w$ at height $k$ by $u_{2}$ (resp. $[k]_{p, q}$, $\left.v[k]_{p, q}\left(1-\delta_{0 k}\right), u_{1}\right)$ if it is $\mathrm{NE}$ (resp. SE, BE, RE) and the weight $P(w)$ of $w$ as the product of weights of its steps. It follows from (4.1) that

$$
B_{n}\left(p, q, u_{1}, u_{2}, v\right)=\sum_{w \in M_{b}(n)} P(w)
$$

Applying a well-known result of Flajolet [6, Propositions 7A and 7B], we derive immediately the continued fraction expansion from the above correspondence.

Proposition 4.1 The generating function $\sum_{n \geq 0} B_{n}\left(p, q, u_{1}, u_{2}, v\right) z^{n}$ has the following continued fraction expansion:

$$
\frac{1}{1-u_{1} z-\frac{u_{2} z^{2}}{1-\left(u_{1}+v\right) z-\frac{u_{2}[2]_{p, q} z^{2}}{1-\left(u_{1}+[2]_{p, q} v\right) z-\frac{u_{2}[3]_{p, q} z^{2}}{1-\left(u_{1}+[3]_{p, q} v\right) z-\frac{u_{2}[4]_{p, q} z^{2}}{\cdots}}}} .}
$$

Note that the $q=v=1$ case of Proposition 4.1 has been given by Biane [1]. Taking $u_{1}=u_{2}=v=1$, we have:

Corollary 4.2 The generating function

$$
\sum_{n \geq 0}\left(\sum_{\pi \in \Pi_{n}} p^{\operatorname{cr}(\pi)} q^{n e(\pi)}\right) z^{n}=\sum_{n \geq 0}\left(\sum_{\pi \in \Pi_{n}} q^{\operatorname{cr}(\pi)} p^{n e(\pi)}\right) z^{n}
$$

has the following continued fraction expansion:

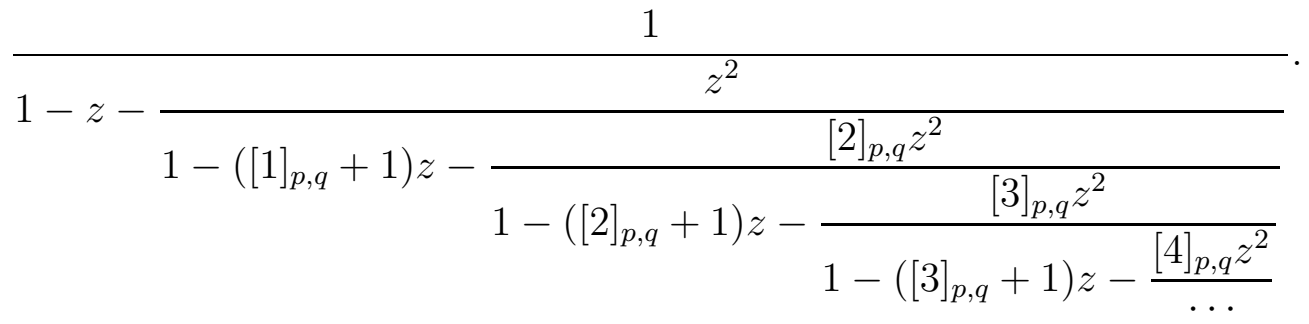


For any $\pi \in \Pi_{n}$, denote by ed $(\pi)$ the number of edges of $\pi$. Clearly we have ed $(\pi)=$ $\mathrm{bl}(\pi)+\operatorname{tr}(\pi)$ and $\operatorname{cr}(\pi)+\operatorname{ne}(\pi)+\operatorname{al}(\pi)=\left(\begin{array}{c}\operatorname{ed}(\pi) \\ 2\end{array}\right)$. Let

$$
E_{n}(v, q):=\sum_{\pi \in \Pi_{n}} q^{\operatorname{cr}(\pi)+\mathrm{ne}(\pi)} v^{e d(\pi)} .
$$

Setting $p=q, u_{1}=1$ and $u_{2}=v$ in Proposition 4.1, we get

Corollary 4.3 The generating function $\sum_{n \geq 0} E_{n}(v, q) z^{n}$ has the following continued fraction expansion:

$$
\frac{1}{1-z-\frac{v z^{2}}{1-(1+v) z-\frac{2 q v z^{2}}{1-(2 q v+1) z-\frac{3 q^{2} v z^{2}}{1-\left(3 q^{2} v+1\right) z-\frac{4 q^{3} v z^{2}}{\cdots}}}} .}
$$

Let $E_{n}(v, q)=\sum_{k \geq 0} e_{k}(q) v^{k}$. Then

$$
F_{n}(q):=\sum_{\pi \in \Pi_{n}} q^{\mathrm{al}(\pi)}=\sum_{k \geq 0} q^{\left(\begin{array}{l}
k \\
2
\end{array}\right)} e_{k}(1 / q) .
$$

Finally consider the enumerating polynomials of crossings and nestings of $\mathcal{M}_{2 n}$ :

$$
L_{n}(p, q)=\sum_{\alpha \in \mathcal{M}_{2 n}} p^{\operatorname{cr}(\alpha)} q^{n e(\alpha)}=\sum_{\alpha \in \mathcal{M}_{2 n}} p^{n e(\alpha)} q^{\operatorname{cr}(\alpha)} .
$$

Setting $u_{2}=1, u_{1}=v=0$ in Proposition 4.1 and replacing $z^{2}$ by $z$ we get

\section{Proposition 4.4}

$$
\sum_{n \geq 0} L_{n}(p, q) z^{n}=\frac{1}{1-\frac{z}{1-\frac{[2]_{p, q} z}{1-\frac{[3]_{p, q} z}{1-\frac{[4]_{p, q} z}{\cdots}}}} .}
$$

Note that the $p=1$ case of Proposition 4.4 corresponds to a result of Touchard [13]. Since a matching of $[2 n]$ has exactly $n$ edges, we get $\operatorname{cr}(\alpha)+\operatorname{ne}(\alpha)+\operatorname{al}(\alpha)=\left(\begin{array}{l}\mathrm{n} \\ 2\end{array}\right)$ for any $\alpha \in \mathcal{M}_{2 n}$. Therefore

$$
T_{n}(q):=\sum_{\alpha \in \mathcal{M}_{2 n}} q^{\mathrm{al}(\alpha)}=q^{\left(\begin{array}{l}
n \\
2
\end{array}\right)} L_{n}(1 / q, 1 / q) .
$$

The first terms of the above sequences are given as follows:

$$
\begin{array}{lll}
T_{0}(q)=T_{1}(q)=1 & L_{0}(p, q)=L_{1}(p, q)=1 \\
T_{2}(q)=2+q & L_{2}(p, q)=1+p+q \\
T_{3}(q)=6+4 q+4 q^{2}+q^{3} & L_{3}(p, q)=1+2 p+2 q+2 p q+p^{2}+q^{2}+2 p^{2} q+2 p q^{2}+p^{3}+q^{3} .
\end{array}
$$




\section{$5 \quad$ Remarks}

Klazar [8] has also a result more general than (1.7), but different from ours. Chen et al [2] have obtained different equinumerous results on crossings and nestings in matchings and partitions, while an analogous result for permutations has been given by Corteel [3]. For a big picture of these results and problems we refer the reader to Krattenthaler's recent paper $[9]$.

\section{References}

[1] Ph. Biane, Some properties of crossings and partitions, Discrete Math., 175(1997), 41-53.

[2] W. Y. C. Chen, E. Y. P. Deng, R. R. X. Du, R. Stanley et C. H. Yan, Crossings and nestings of matchings and partitions, preprint, math.CO/0501230, 2005.

[3] S. Corteel, crossings and alignments of permutations, preprint, math.CO/0505031, 2005.

[4] A. de Médicis et X. Viennot, Moments des q-polynômes de Laguerre et la bijection de Foata-Zeilberger, Adv. Appl. Math., 15 (1994), 262-304.

[5] M. de Sainte-Catherine, Couplage et Pfaffiens en combinatoire, physique et informatique, Thèse du 3me cycle, Université de Bordeaux I, 1983.

[6] Ph. Flajolet, Combinatorial aspects of continued fractions, Discrete Math. 41 (1982), 145153.

[7] J. Françon and X. Viennot, Permutations selon les pics, creux, doubles descentes, doubles montées, Discrete Math. 28 (1979), 21-35.

[8] M. Klazar, On identities concerning the numbers of crossings and nestings of two edges in matchings, preprint, math.CO/0510676, 2005.

[9] C. Krattenthaler, Growth diagrams, and increasing and decreasing chains in fillings of Ferrers shapes, preprint, math.CO/0503012, 2005.

[10] G. Ksavrelof and J. Zeng, Nouvelles statistiques de partitions pour les $q$-nombres de Stirling de seconde espèce, Discrete Math, 256 (2002), 743-758.

[11] R. Simion, Non crossing partitions, Discrete Math, 217 (2000), 367-409.

[12] R. Stanley, Enumerative Combinatorics II, Cambridge Studies in Advanced Mathematics, 1999.

[13] J. Touchard, Sur un problème de configurations et sur les fractions continues, Canad. J. Math, 4 (1952), 2-25.

[14] X. Viennot, Une théorie combinatoire des polynômes orthogonaux, Lecture Notes, UQAM, Montréal 1983. 